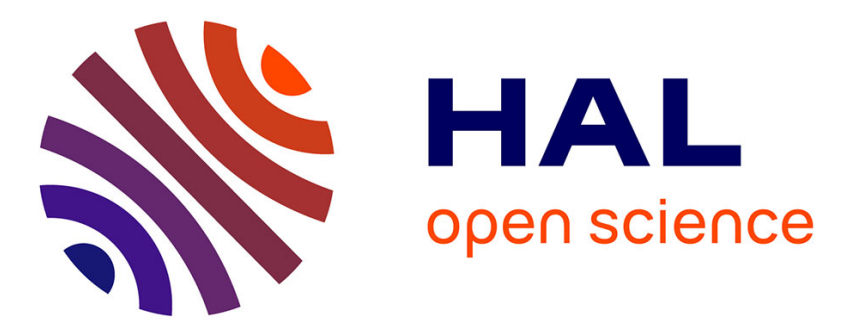

\title{
Synthesis and application of molecularly imprinted polymers for the selective extraction of organophosphorus pesticides from vegetable oils
}

Sara Boulanouar, Audrey Combes, Sakina Mezzache, Valérie Pichon

\section{To cite this version:}

Sara Boulanouar, Audrey Combes, Sakina Mezzache, Valérie Pichon. Synthesis and application of molecularly imprinted polymers for the selective extraction of organophosphorus pesticides from vegetable oils. Journal of Chromatography A, 2017, 1513, pp.59-68. 10.1016/j.chroma.2017.07.067 . hal-01628409

\section{HAL Id: hal-01628409 \\ https://hal.sorbonne-universite.fr/hal-01628409}

Submitted on 3 Nov 2017

HAL is a multi-disciplinary open access archive for the deposit and dissemination of scientific research documents, whether they are published or not. The documents may come from teaching and research institutions in France or abroad, or from public or private research centers.
L'archive ouverte pluridisciplinaire HAL, est destinée au dépôt et à la diffusion de documents scientifiques de niveau recherche, publiés ou non, émanant des établissements d'enseignement et de recherche français ou étrangers, des laboratoires publics ou privés. 
Journal of Chromatography A, 1513, 2017, 59-68, https://doi.org/10.1016/j.chroma.2017.07.067

\title{
Synthesis and application of molecularly imprinted polymers for the selective extraction of organophosphorus pesticides from vegetable oils
}

\author{
Sara Boulanouar ${ }^{a}$, Audrey Combès ${ }^{a}$, Sakina Mezzache ${ }^{b}$, Valérie Pichon ${ }^{a, c}$ \\ a Dept of Analytical, Bioanalytical Sciences and Miniaturization (LSABM) - UMR CBI 8231 \\ (CNRS-ESPCI), ESPCI, PSL Research University, 10 rue Vauquelin, 75231 Paris Cedex 05, France. \\ b \\ L'Oréal Research and Innovation, 1 avenue Eugène Schueller BP22, 93601 Aulnay sous Bois \\ Sorbonne Universités, UPMC, 4 Place Jussieu, 75005 Paris \\ Corresponding author: Valérie Pichon, valerie.pichon@espci.fr, +33140794772 \\ Corresponding author institution: LSABM-ESPCI, 10 rue Vauquelin, 75005 Paris, France.
}

\section{Abstract}

The increasing use of pesticides in agriculture causes environmental issues and possible serious health risks to humans and animals. Their determination at trace concentrations in vegetable oils constitutes a significant analytical challenge. Therefore, their analysis often requires both an extraction and a purification step prior to separation with liquid chromatography (LC) and mass spectrometry (MS) detection. This work aimed at developing sorbents that are able to selectively extract from vegetable oil samples several organophosphorus (OPs) pesticides presenting a wide range of physico-chemical properties. Therefore, different conditions were screened to prepare molecularly imprinted polymers (MIPs) by a non-covalent approach. The selectivity of the resulting polymers was evaluated by studying the OPs retention in pure media on both MIPs and non-imprinted polymers (NIP) used as control. The most promising MIP sorbent was obtained using monocrotophos (MCP) as the template, methacrylic acid (MAA) as the monomer and ethylene glycol dimethacrylate (EGDMA) as the cross-linker with a molar ratio of $1 / 4 / 20$ respectively. The repeatability of the extraction procedure and of the synthesis procedure was demonstrated in pure media. The capacity of this MIP was $1 \mathrm{mg} / \mathrm{g}$ for malathion. This MIP was also able to selectively extract three OPs from almond oil by applying the optimized SPE procedure. Recoveries were between 73 and $99 \%$ with SD values between 4 and $6 \%$ in this oil sample. The calculated LOQs (between 0.3 and $2 \mu \mathrm{g} / \mathrm{kg}$ ) in almond seeds with a SD between 0.1 and $0.4 \mu \mathrm{g} / \mathrm{kg}$ were lower than the Maximum Residue Levels (MRLs) established for the corresponding compounds in almond seed.

Keywords: molecularly imprinted polymers; organophosphorus pesticides; solid phase extraction; vegetable oils; liquid chromatography; mass spectrometry.

\section{Introduction}

Vegetable oils occupy a large place among food products and their nutritional or health contribution does no need demonstration. Their constituents play a very important role in 
human health. In addition, their beneficial properties in cosmetics have been known since antiquity by nourishing, protecting and moisturizing the skin. However, pesticides used in agriculture may possibly be found in vegetable oils. The EU harmonization of the pesticides Maximum Residue Levels (MRLs) within Regulation 396/2005 has led to specific MRLs being set on raw materials (oil seeds and oil fruit), but not on processed products. A processing factor was proposed by FEDIOL (vegetable oil and protein meal industry association), to define the limits allowed in the processed products such as vegetable oils, fats and meals. To reach the MRLs values that are established at $10 \mu \mathrm{g} / \mathrm{kg}$ by EU for pesticides as general default for food or feed constitutes a significant analytical challenge for the safe use of such oils.

Organophosphorus (OPs) compounds constitute an important class of pesticides whose toxicity arises from the inhibition of the acetylcholinesterase enzyme. They exhibit a wide range of physicochemical properties thus rendering their determination in complex oil samples particularly difficult. Their analysis often requires a previous extraction step using gel permeation [1] or a liquid-liquid extraction (LLE) step [2] that is nowadays usually followed by a purification step by dispersive solid-phase (dSPE) extraction, i.e. a global QuEChERSbased procedure adapted for fatty matrices [3-5]. Primary secondary amine (PSA), octadecylsilica (C18) and graphitized carbon black (GCB) are the three most commonly used sorbents for QuEChERS. However, their amount and their proportion when they are used in combination must be optimized to reach the most powerful clean-up effect without affecting the extraction recovery of the target analytes [5]. Indeed, it was recently shown that the addition of GCB to PSA/C18 was efficient for trapping oil components and their removal from the extract but also affects the extraction recovery for some compounds [3].

These drawbacks led to the recent development of molecularly imprinted polymers (MIPs). These synthetic polymeric materials possess specific cavities designed for a template molecule involving a retention mechanism based on molecular recognition. The MIPs have been already successfully used in several fields, such as sensors, organic synthesis and separation of enantiomers [6-9]. The first application of a MIP as SPE sorbent was carried out by Sellergren et al. in 1994 for extracting pentamidine present at low concentrations in urine [10]. The principle of selective extraction on a MIP is the same as for a conventional SPE sorbent. After a conditioning step, the sample is percolated through the MIP and a washing step removes the interfering compounds. The desorption of analytes is achieved by percolating a solvent able to develop interactions with the sorbent in order to desorb the analytes retained on the MIP. Several MIPs dedicated to the selective extraction of mycotoxins, drugs, pollutants or steroids are now commercially available.

The development of MIPs for the extraction of OPs has been largely reported these last years. MIPs were prepared as particles to be used in cartridges between two frits as SPE sorbent [11-26] or as dispersive sorbent for dSPE [27-31] and for matrix solid-phase dispersion (MSPD) [32-34] or as a thin film in solid-phase microextraction (SPME) [35-37] or in stir bar sorption extraction (SBSE) [38]. They were applied to the selective extraction of OPs from vegetable extracts (cucumber, lettuce, apple, pear...) and environmental samples such as waters and soil extracts.

In the common approach, the synthesis of MIPs involves first the complexation of a template molecule with functional monomers through non-covalent bonds in a porogenic solvent, followed by polymerization of these monomers around the template with the help of a cross-linker in the presence of an initiator. The choice of the chemical reagents used for the synthesis of the MIP must be judicious in order to really create specific cavities designed for 
the template molecule. In $85 \%$ of the reported works, MIP for OPs were produced in fixed conditions without optimizing the nature and the ratio of the reagents. The target OP was taken as template molecule, methacrylic acid as monomer, ethylene glycol dimethacrylate as cross-linker in a non protic solvent (mainly acetonitrile, dichloromethane and chloroform). The effect of the template was studied only once for the development of an MIP for dimethoate and its metabolite omethoate showing that the metabolite was better adapted for the trapping of both molecules [19]. A few studies described the synthesis of an MIP by varying the nature of the monomer $[11,19,28,30,31]$ and/or the porogen $[11,25,30,31]$ or the template/monomer ratio [24]. In some studies, the choice of the monomer for a given template resulted from studies by molecular modeling and computational design $[17,19,23]$. Once, the MIP synthesized, its selectivity was mainly evaluated by binding experiments or retention studies in pure media. These evaluations were achieved by comparing results using the MIP with results obtained using a non-imprinted polymer (NIP) that is prepared in the same conditions as MIP but in the absence of template. In most reported works, these studies were carried out using up to three OPs including the OP used as template. This comparison between MIP and NIP achieved in spiked pure media allows to put in evidence the presence of cavities in the MIP and is also useful to optimize the extraction procedure that must give rise to high extraction recovery on the MIP and low one on the NIP [8]. Except for one reported work [21], the conditions of extraction finalized in pure media were applied to real samples $[11,17-20,22-27,38]$ without a control of the selectivity by using the NIP or without reoptimization of the extraction conditions to circumvent matrix effects as already reported [8]. These matrix effects were well illustrated by Sanagi et al. who reported recoveries obtained in pure media and in real samples after applying the same extraction procedure on MIP and on NIP [21]. While recovery of extraction for quinalphos in pure media was $92.3 \%$ and $43.9 \%$ for MIP and NIP respectively, the recovery was $99 \%$ and $64.8 \%$, respectively for a real sample, thus illustrating the effect of the matrix components that increase the retention on both sorbents and induce consequently a loss of selectivity. At last, very few works reported the application of MIPs for the selective extraction of OPs from vegetable oils. These works were carried out by Bakas et al. who studied the extraction of methidathion [17], dimethoate [19] and fenthion [23] from olive oil samples.

The objectives of this work were to prepare a MIP able to extract from vegetable oil the maximum number of OPs that were selected by taking into account the risk of their occurrence in such samples. For this, different conditions of synthesis were screened by varying the nature of the template, of the monomer and of the porogenic solvent in order to find the conditions of synthesis of a MIP able to selectively trap the largest number of OPs from vegetable oils The MIP resulting in best selectivity for five OPs was studied more in detail by investigating its behavior towards ten OPs from pure media but also from vegetable oils whose content may affect the recoveries on the MIP. At last, to highlight the potential of the developed MIP, a comparison with results obtained while applying C18 silica to an almond oil extract was performed.

\section{Materials and methods}

\subsection{Chemicals}

HPLC-grade acetonitrile $(\mathrm{ACN})$, methanol $(\mathrm{MeOH})$, dichloromethane $(\mathrm{DCM})$ and 
toluene were supplied by Carlo Erba (Val de Reuil, France). High purity water was dispensed by a Milli-Q purification system (Millipore, Saint Quentin en Yvelines, France).

Certified reference material : dimethoate (DMT) 98\%, fenthion sulfoxide (FSX) 99\%, fenthion sulfone (FSN) 99\%, methidathion (MTH) 98\%, malathion (MAL) 99\%, fenitrothion (FNT) 98\%, diazinon (DIZ) 98\%, pirimiphos methyl (PIM) 99.5\%, fenthion (FEN)99\% and chlorpyrifos-ethyl (CLE) 99.5\% were supplied by Cluzeau Info Labo (Saint-Foy-La-Grande, France). Individual stock solutions from each OP were made at a concentration of $100 \mathrm{mg} / \mathrm{L}$ in ACN. A stock solution mixture containing $5 \mathrm{mg} / \mathrm{L}$ of each OP was prepared in ACN and stored at $4{ }^{\circ} \mathrm{C}$ until further use.

Parathion ethyl (PE), monocrotophos (MCP), fenamiphos (FEM), 2-trifluoromethyl acrylic acid (TFMA) 98\%, acetonitrile anhydrous 99.8\%, ammonium acetate for HPLC $99.0 \%$ $(A C)$, n-hexane, methacrylic acid (MAA) and ethylene glycol dimethacrylate (EGDMA) were supplied by Sigma-Aldrich (Saint Quentin Fallavier, France). Washed EGDMA and MAA were distilled under vacuum in order to remove inhibitors. Azo- $N, N^{\prime}$-bis-isobutyronitrile (AIBN) was purchased from Acros Organics (Noisy-le-Grand, France). Acetic and formic acids (AA and FA respectively) were purchased from VWR (Fontenay-sous-Bois, France).

\subsection{Apparatus and analytical conditions}

The LC-MS/MS analyses were performed using a liquid chromatograph (UltiMate $3000^{\circ}$, Thermo Scientific, Illkirch, France) coupled with Triple Stage Quadrupole Mass Spectrometer (TSQ Quantum Access MAX, Thermo Scientific, Illkirch, France) equipped with a heated electrospray ionization source (HESI2). The chromatographic separation was performed on Accucore PFP column $(150 \times 2.1 \mathrm{~mm}, 2.6 \mu \mathrm{m}$, ThermoFisher Scientific, Villebon Courtaboeuf France) maintained at $32{ }^{\circ} \mathrm{C}$ with a column oven (Croco-cil, Interchim). Samples were analysed using linear gradient elution with water containing $0.1 \%(\mathrm{v} / \mathrm{v})$ of $F A$ and $4 \mathrm{mM}$ of $\mathrm{AC}(\mathrm{A})$ and $\mathrm{MeOH}$ containing $0.1 \%(\mathrm{v} / \mathrm{v})$ of $\mathrm{FA}$ and $4 \mathrm{mM}$ of $\mathrm{AC}(\mathrm{B})$. The gradient started at $20 \%$ of $B$ during $2.5 \mathrm{~min}$ and increased to $80 \%$ of $B$ in $23.5 \mathrm{~min}$, held for $2 \mathrm{~min}$, and returned to initial composition within $2 \mathrm{~min}$ and let $2 \mathrm{~min}$ to equilibrate the system. The flow rate was set at $0.4 \mathrm{~mL} / \mathrm{min}$ and the injection volume was $2 \mu \mathrm{L}$.

For the capacity study, the LC gradient was shorter. This new gradient started with an equilibration during 2 minutes with $20 \%$ of B and increased to $80 \%$ in 5 min, held for 3 min, and returned to initial composition within $2 \mathrm{~min}$ and let $2 \mathrm{~min}$ to equilibrate the system.

MS was operated in positive ion mode with MRM detection using an electrospray voltage of $3500 \mathrm{~V}$ and a skimmer offset of $5 \mathrm{~V}$. Capillary and vaporizer temperatures were set at $280{ }^{\circ} \mathrm{C}$ and $295{ }^{\circ} \mathrm{C}$ respectively. Sheath gas pressure and auxiliary gas pressure were set respectively at 55 and 15 units. Nitrogen was used as nebulizer and desolvatation gas and argon as the collision gas at a pressure of 1.5 mTorr. For the optimization of the MS detection, each OP was infused at a concentration of $5 \mathrm{mg} / \mathrm{L}$ in the mixture $A / B(50 / 50, \mathrm{v} / \mathrm{v})$. The quantification of 10 the OPs was performed in MRM mode using the specific transitions FEN and FNT both gave a very low signal intensity during infusion. A second transition was used for confirmation purposes and to avoid false positive responses. The $\mathrm{m} / \mathrm{z}$ values, tube lens and collision energies values corresponding to quantitation and confirming ions were summarized in the supplementary material 1 (table S1).

The LC-DAD analyses were performed using a liquid chromatograph (LC) Agilent 1200 series (Agilent Technology, Massy, France) system equipped with a binary pump, an auto 
sampler and a diode array detector (DAD) controlled by a Chemstation software. OPs were separated using the same column, flow rate and injection volume as for LC-MS/MS analysis. Samples were analysed using linear gradient elution with water $(A)$ and ACN (B). The gradient started with $8 \%$ of B during $2.5 \mathrm{~min}$ and increased to $60 \%$ in $23.5 \mathrm{~min}$, held for $2 \mathrm{~min}$, returned to initial composition within $2 \mathrm{~min}$ and let $2 \mathrm{~min}$ to equilibrate the system. DMT, MTH, MAL were quantified at $210 \mathrm{~nm}, \mathrm{FSX}$ at $240 \mathrm{~nm}, \mathrm{FSN}$ at $230 \mathrm{~nm}, \mathrm{FNT}$ at $270 \mathrm{~nm}, \mathrm{DIZ}, \mathrm{PIM}$ and FEN at $250 \mathrm{~nm}$ and CLE at $290 \mathrm{~nm}$.

\subsection{Synthesis of the MIPs}

MIPs were synthesized as bulk using a non-covalent approach. Different combinations of templates; monomers and solvents were tested (Table 1). A template/monomer/crosslinker molar ratio of $1 / 4 / 20$ was used for all syntheses. Briefly $0.25 \mathrm{mmol}$ of template and 1 $\mathrm{mmol}$ of monomer were dissolved in $1.4 \mathrm{~mL}$ of solvent in a glass tube (14 $\mathrm{mm}$ i.d.). Then, 5 $\mathrm{mmol}$ of the cross-linker (EDGMA) and $10 \mathrm{mg}$ of the initiator AIBN, were added to the mixture and purged by nitrogen for $10 \mathrm{~min}$. The tube was sealed and placed in a water bath at $60^{\circ} \mathrm{C}$ for $24 \mathrm{~h}$. A non-imprinted polymer was simultaneously prepared in the same conditions but without adding the template. Each obtained polymer was crushed, ground automatically in a mixer MIL MM 301 from Retsch ${ }^{\circledR}$ at $35 \mathrm{~s}^{-1}$ for $3 \times 1 \mathrm{~min}$ and sieved in a vibratory sieve shaker from Retsch ${ }^{\circledR}$ using amplitude of $15 \mathrm{~mm} / \mathrm{g}$ for $5 \mathrm{~min}$. The particles sizes between 25 and 36 $\mu \mathrm{m}$ were collected and a sedimentation with $4 \times 5 \mathrm{~mL}$ of $\mathrm{MeOH} /$ water 80/20, v/v was performed to remove the thin particles and then dried $24 \mathrm{~h}$ at room temperature.

After that, between 25 and $35 \mathrm{mg}$ of polymer were packed in a $1 \mathrm{~mL}$ disposable cartridge of propylene (Interchim) between two polyethylene frits ( $20 \mu \mathrm{m}$, Sigma-Aldrich). The polymer was washed with $\mathrm{MeOH}$ (approximately $10 \mathrm{~mL}$ ) containing $10 \%$ of AA $(\mathrm{v} / \mathrm{v})$. The washing fractions were evaporated and suspended in $\mathrm{MeOH}, \mathrm{ACN}$ and $\mathrm{H}_{2} \mathrm{O}(40 / 10 / 50, \mathrm{v} / \mathrm{v} / \mathrm{v})$ for the MCP template, and in ACN for the other templates before injection in LC/UV. The polymers were washed until the template could no longer be detected in the washing fraction by LC-UV at $210 \mathrm{~nm}$ for MCP, $250 \mathrm{~nm}$ for DIZ and F, and $280 \mathrm{~nm}$ for PE. Then the cartridge was washed with $10 \mathrm{~mL}$ of $\mathrm{MeOH}$ to remove residual $\mathrm{AA}$.

Table 1: Conditions of the synthesis of six MIPs, using AIBN as initiator and a molar ratio template /monomer/cross-linker of 1/4/20. NIPs were synthetized in the same conditions without introducing the template. MAA: methacrylic acid, EGDMA: ethylene glycol dimethacrylate, TFMA: 2(trifluoromethyl) acrylic acid, DCM: dichloromethane, ACN: acetonitrile.

\begin{tabular}{ccccc}
\hline Sorbent & Template & Monomer & Cross-linker & Porogen \\
\hline MIP 1 & PE & MAA & EGDMA & DCM \\
MIP 2 & MCP & MAA & EGDMA & DCM \\
MIP 3 & F & MAA & EGDMA & DCM \\
MIP 4 & DIZ & MAA & EGDMA & DCM \\
MIP 5 & DIZ & MAA & EGDMA & ACN \\
MIP 6 & DIZ & TFMA & EGDMA & ACN \\
\hline
\end{tabular}




\subsection{SPE procedure applied in pure media}

Different studies were carried out on the synthesized polymers to optimize the SPE procedure, as the selection of the percolation solvent or the washing solution. Before the percolation, the cartridges were conditioned with $4 \mathrm{~mL}$ of the used percolation solvent. Then $1 \mathrm{~mL}$ of percolated solvent (toluene, DCM, hexane or mix of hexane, DCM and ACN (70/29/1, $\mathrm{v} / \mathrm{v} / \mathrm{v})$ ) spiked with $1 \mathrm{mg} / \mathrm{L}$ of PE was passed through MIP/NIP 1 cartridges. To study the washing solvents, a spiked solution of hexane using six OPs at $1 \mathrm{mg} / \mathrm{L}$ was used as percolation solution on the 6 synthesized MIPs/NIPs. Three washing steps were included in an SPE procedure: $1 \mathrm{~mL}$ of hexane and DCM (80/20, v/v), $1 \mathrm{~mL}$ of hexane, DCM and ACN (80/18/2, $\mathrm{v} / \mathrm{v} / \mathrm{v})$ and $1 \mathrm{~mL}$ of hexane, DCM and ACN (80/15/5, v/v/v). The second procedure applied to the six synthesized MIPs consisted in a single washing step with $1 \mathrm{~mL}$ of a mixture of hexane and $\operatorname{DCM}(95 / 5, v / v)$. After the washing step, the cartridge was dried by $5 \mathrm{~mL}$ of air. Finally, the OPs were eluted with $1 \mathrm{~mL}$ of ACN. Each fraction resulting from each step was evaporated to dryness by a nitrogen stream and was resuspended in $0.5 \mathrm{~mL}$ of $A C N$ before injection in the LC-DAD system.

\subsection{Extraction of OPs from the vegetable oils}

\subsubsection{Preliminary extraction procedure for the vegetable oils}

Before the SPE procedure using MIP or NIP sorbents, an LLE was performed on oil samples. This LLE procedure was described by the ITERG (French Institute specialized in fats and oils) and used before an SPE step using a C18 sorbent. LLE was carried out using $3 \times 1 \mathrm{~mL}$ of a mixture of ACN and DCM (90/10, v/v) for $200 \mathrm{mg}$ of oil. The obtained oil extract was evaporated to dryness under nitrogen stream and was spiked with $2.5 \mathrm{mg} / \mathrm{kg}$ of ten OPs in $1 \mathrm{~mL}$ of hexane and passed through the MIP 2 and NIP 2 cartridges. After a conditioning step with $4 \mathrm{~mL}$ of hexane, the oil extract was percolated and $1 \mathrm{~mL}$ of a mixture of hexane and DCM $(95 / 5, v / v)$ was used for the washing step. Finally, the OPs were eluted in $1 \mathrm{~mL}$ of ACN. The elution fraction was directly injected in the LC-MS/MS and LC-UV systems. For the clean-up on $\mathrm{C} 18,12 \mathrm{~mL}$ of $\mathrm{MeOH}$ and $12 \mathrm{~mL}$ of $\mathrm{ACN}$ were passed through the cartridge for conditioning, then $3 \mathrm{~mL}$ of oil extract resulting from the LLE step were percolated, and $1.5 \mathrm{~mL}$ of $\mathrm{MeOH}$ was used for the elution step. The elution fraction was recollected and evaporated under nitrogen stream. Finally, the dry extract was suspended in ACN, before its analysis by LC-MS.

\subsubsection{Optimized extraction procedure on MIP for the vegetable oils}

Optimization of the SPE procedure was necessary to reach the MRLs established by the regulation (EC) No 396/2005 of the European Parliament and of the Council of 23 February 2005. LLE was carried out as in the previous section. The obtained oil extract was evaporated to dryness under nitrogen stream, diluted with $10 \mathrm{~mL}$ of hexane and was spiked with a low concentration of $100 \mu \mathrm{g} / \mathrm{kg}$ of three OPs (MTH, MAL, DIZ). After conditioning the MIP/NIP with $4 \mathrm{~mL}$ of hexane, $1 \mathrm{~mL}$ of the oil extract was percolated through MIP/NIP cartridges and different volumes of washing solution hexane and DCM $(95 / 5, \mathrm{v} / \mathrm{v})$ were tested: $0.4,0.65,0.8$ and $1 \mathrm{~mL}$. Finally, the OPs were eluted with $1 \mathrm{~mL}$ of ACN. The elution fraction was evaporated to dryness under nitrogen stream and suspended in $100 \mu \mathrm{L}$ of ACN before injection in the LCMS/MS system. 


\subsection{Study of the capacity}

The study of the capacity of the MIP was performed using percolation solutions that contained different amounts (between 0.5 and $87 \mu \mathrm{g}$ ) of MAL in $1 \mathrm{~mL}$ of hexane through the MIP and NIP cartridges. Before percolation, the cartridges were conditioned with $4 \mathrm{~mL}$ of hexane. Then, for the washing step, $1 \mathrm{~mL}$ of hexane and DCM $(95 / 5, \mathrm{v} / \mathrm{v})$ was passed through the cartridge. Finally, the OPs were eluted with $1 \mathrm{~mL}$ of ACN. The elution fractions were diluted with ACN taking into account the linearity range of MAL $(10-250 \mu \mathrm{g} / \mathrm{L})$ and were directly injected in LC-MS/MS using the specific transition $(348 \rightarrow 127)$ for the quantitation of MAL.

\section{Results and discussions}

\subsection{Development of the LC-UV and LC-MS analyses}

To ensure a good quantification of the OPs, the development of an analytical separation was necessary. Taking into account the hydrophobicity of the studied OPs (see Figure 1), different non-polar columns were tested using different linear gradient modes. The first column, Atlantis C18 (150 x $2.1 \mathrm{~mm}, 3.5 \mu \mathrm{m}$, Waters), was not able to separate PE and FEN. Hence, a fused-core column Zorbax Poroshell 120 EC-C18 (50 x 2.1 mm, $2.7 \mu \mathrm{m}$, Agilent), was tested but a low resolution was obtained for DIZ and FEN. A third column, Accucore RPMS 120 EC-C18 (100 x $2.1 \mathrm{~mm}, 2.6 \mu \mathrm{m}$, Thermoscientific) yielded a better resolution for DIZ and FEN, but the separation of MAL and FNT was not possible with this column. Finally, the best separation was obtained using an Accucore PFP column $(150 \times 2.1 \mathrm{~mm}, 2.6 \mu \mathrm{m}$, Thermoscientific). The LOQ values (defined as the concentration level that gives a signal to noise ratio $\mathrm{S} / \mathrm{N}$ of 10 ) ranged from 30 to $300 \mu \mathrm{g} / \mathrm{L}$ depending on the OPs (Table 2) using the LC-UV conditions described in Section 2.2. These values of LOQ allowed the OPs to being quantified and the performance of the MIPs to being evaluated in pure media. However, for the studies related to the application of the MIPs to oil samples, it was necessary to develop and to use the more sensitive LC-MS/MS method in MRM mode in order to decrease the LOQs (operating conditions described in Section 2.2). The obtained LOQs are summarized in Table 2 , and range from 0.4 to $7 \mu \mathrm{g} / \mathrm{L}$ for the OPs in pure media, with the exception of FEN whose, the estimated LOQ was $1000 \mu \mathrm{g} / \mathrm{L}$.

Table 2. Comparison of LODs $(S / N=3)$ and LOQs $(S / N=10)$ in $\mu \mathrm{g} / \mathrm{L}$ obtained in LC-UV and LC- MS/MS and estimated by injecting OPs at $200 \mu \mathrm{g} / \mathrm{L}$ in LC-UV and at $5 \mu \mathrm{g} / \mathrm{L}$ in LC-MS (except for FEN, $1000 \mu \mathrm{g} / \mathrm{L}$ ).

\begin{tabular}{ccccc}
\hline Compounds & \multicolumn{2}{c}{ LC-UV } & \multicolumn{2}{c}{ LC-MS } \\
\hline OPs & LOD & LOQ & LOD & LOQ \\
\hline DMT & 50 & 160 & 0.6 & 2.2 \\
FSX & 20 & 70 & 0.1 & 0.4 \\
FSN & 2 & 10 & 2.1 & 6.9 \\
MTH & 50 & 170 & 0.3 & 0.9 \\
MAL & 90 & 300 & 0.2 & 0.8 \\
DIZ & 50 & 160 & 0.08 & 0.3 \\
FNT & 20 & 50 & No signal & No signal \\
FEN & 10 & 30 & 300 & 1000 \\
PIM & 20 & 60 & 0.2 & 0.8 \\
CLE & 30 & 90 & 0.4 & 1.3 \\
\hline
\end{tabular}




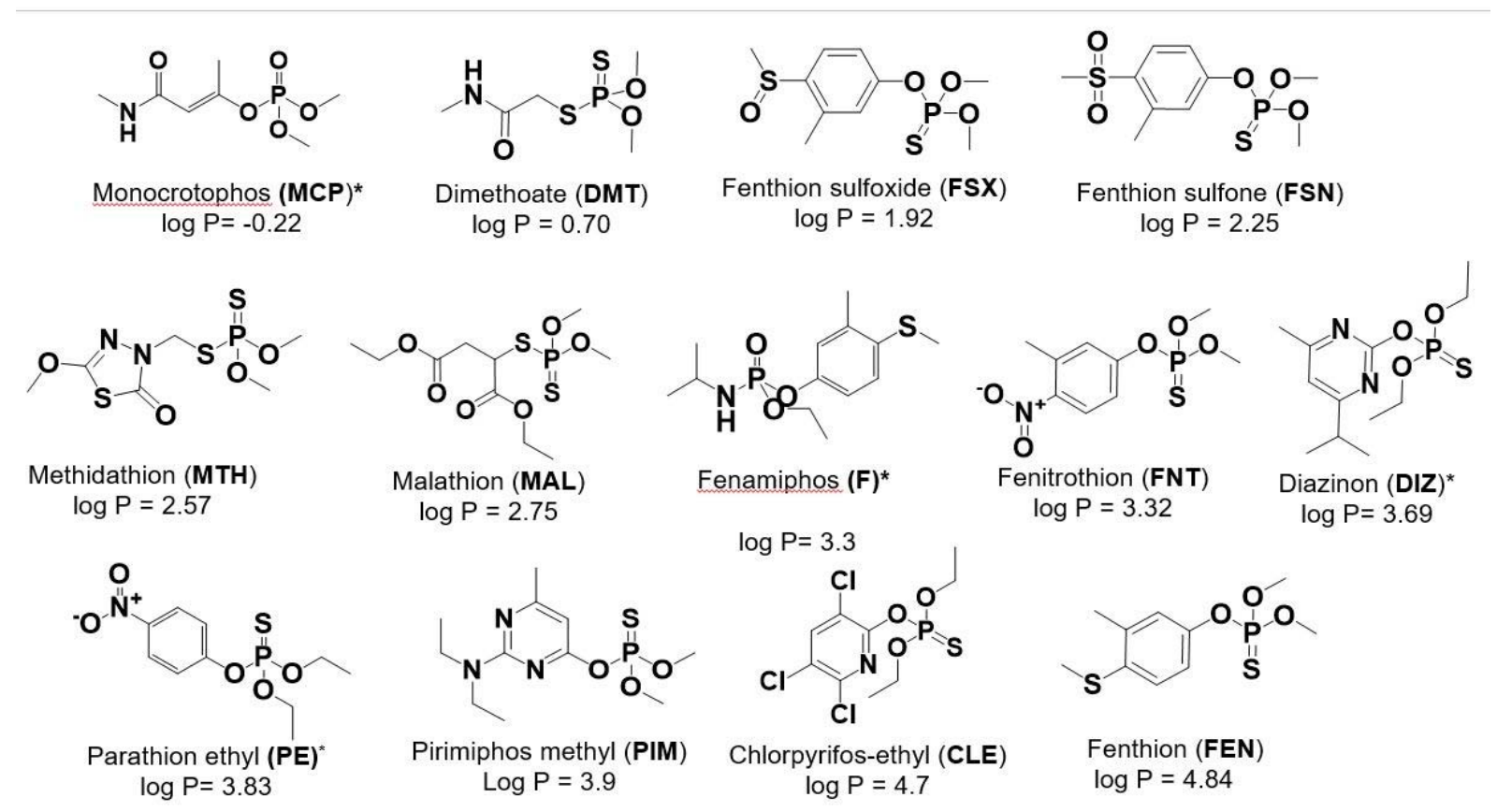

Figure 1: Chemical structure and partition coefficient of ten OPs and of the templates*. Log P values are issued from Pesticide Properties DataBase from University of Hertfordshire.

\subsection{Screening of the synthesis conditions}

\subsubsection{Choice of the MIP synthesis conditions}

Several synthesis conditions were screened in order to determine which ones resulted for the largest number of OPs in the highest selectivity during the extraction procedure, i.e a low retention of OPs on the NIP and a strong retention on the MIP. The non-covalent approach was selected because it is the most common one used to prepare MIPs for SPE [40]. The synthesis of MIPs involves first the complexation of a template molecule with a functional monomer, through non-covalent bonds, followed by polymerization of this monomer around the template with the help of a cross-linker and in the presence of an initiator [41]. Finally, the template molecule is removed from the highly cross-linked polymer, thus leaving specific cavities complementary to the template in shape, size and functionality.

Target analytes were selected by taking into account the most frequently detected OPs in different vegetable oils by ITERG. As these OPs presented a large structural variety and a broad range of octanol-water partition coefficients (Figure 1), different templates were used to prepare the MIPs (Table 1). PE was selected for the MIP 1 synthesis because it is an analogue of FNT. MCP was used for the MIP 2 synthesis because it has a linear structure like DMT and MAL. F was selected for the MIP 3 synthesis because it presents similarities, namely the benzyl and the phosphate groups with FEN, FSX and FSN. Finally DIZ, although it is also a target OP, was used for the MIP 4 synthesis because it presents similar heterocycles and a thiophosphoric $(\mathrm{P}=\mathrm{S})$ group as $\mathrm{CLE}, \mathrm{PIM}$ and $\mathrm{MTH}$. The choice of the functional monomer also constitutes one of the most important factors governing the properties of MIPs. MAA was used for MIPs 1 to 5 and TFMA for MIP 6. These monomers were selected because the OPs include nitrogen, oxygen and sulfur atoms that can form electrostatic interactions with these acidic monomers or hydrogen bonds. In order to enhance this type of interactions, slightly polar and non-protic 
solvents, DCM (MIP 1 to 5) or ACN (MIP 5) were tested. To obtain a highly cross-linked structure, an excess of cross-linker, EGDMA, was added to the polymerization mixture. Once the six MIP/NIPs were synthesized, the optimization of the SPE procedure was necessary to evaluate the performance of these supports.

\subsubsection{Choice of the percolation solvents}

In order to select the solvent favoring the retention of OPs on the synthesized MIPs, a preliminary experiment was carried out by percolating different solvents spiked with one OP on one MIP only, MIP 1, the nature of the expected interaction between the OPs and the MIPs being similar, i.e. polar interactions. PE was selected for this experiment, as it is one of the selected templates. To favor the specific interactions between the monomer and the target compounds during the percolation step, solvents with low polarities (toluene, DCM, hexane and a mix of hexane, DCM and ACN (70/29/1, v/v/v)) spiked with $1 \mathrm{mg} / \mathrm{L}$ of PE were passed through MIP/NIP 1 cartridges. PE was not retained during the percolation step in toluene, and was weakly retained in DCM (50\%) and in the mixture of hexane, DCM and ACN (70/29/1, $\mathrm{v} / \mathrm{v} / \mathrm{v})(50 \%)$, whereas using hexane as percolation solvent, the retention was strong on MIP and on NIP. Therefore, hexane was selected as the solvent of percolation to evaluate the retention on other MIPs.

\subsubsection{Comparison of the synthesized MIPS}

To favor the selectivity brought by the MIPs, the washing step was optimized to decrease the retention on NIPs (that is caused by non-specific interactions at the surface of the polymer) while maintaining a high retention on the MIPs by specific interactions that should take place in their cavities. For this experience, and to limit data treatment, the retention of six OPs among the ten was studied on the six synthesized MIPs/NIPs (Table 1). These OPs (FSX, MAL, DIZ, FNT, FEN and CLE) were selected according to their polarity, from one of the most to the less polar (FSX, FEN respectively) and by adding four other OPs of intermediate polarities in order to cover the whole range of polarity. The cartridges were conditioned first with $4 \mathrm{~mL}$ of hexane, then $1 \mathrm{~mL}$ of hexane spiked with $1 \mathrm{mg} / \mathrm{L}$ of six OPs was percolated on each MIP/NIP. Three successive washing steps were applied: $1 \mathrm{~mL}$ of hexane and DCM (80/20, v/v) (W1), $1 \mathrm{~mL}$ of hexane, DCM and ACN (80/18/2, v/v/v) (W2) and then $1 \mathrm{~mL}$ of hexane, DCM and ACN $(80 / 15 / 5, \mathrm{v} / \mathrm{v} / \mathrm{v})(\mathrm{W} 3)$, the augmentation of the polarity of the mixture increasing its elution strength. As observed for $\mathrm{PE}$, most of the six OPs were retained during the percolation step, but more than $70 \%$ were lost during the first washing step (W1) from the six MIPs/NIPs. However, CLE was not retained (loss during percolation step) on the six MIPs/NIPs because it was not able to develop strong polar interactions with the MIP. To optimize the selectivity for the retained OPs, a washing solution of a lower elution strength was tested by introducing only $5 \%$ of DCM in hexane $(1 \mathrm{~mL})$. The elution step was ensured with a more polar solvent, i.e acetonitrile. In these conditions, a strong retention of the studied compounds was obtained but without any selectivity for MIP 5 and MIP 6. Indeed, similar extraction profiles were obtained on MIP 5 and NIP 5 and on MIP 6 and NIP 6 . Therefore, these two supports were removed from the study. For the four other MIPs/NIPs, the recovery of the five OPs in the elution fraction by applying this extraction procedure is reported on Figure 2. 


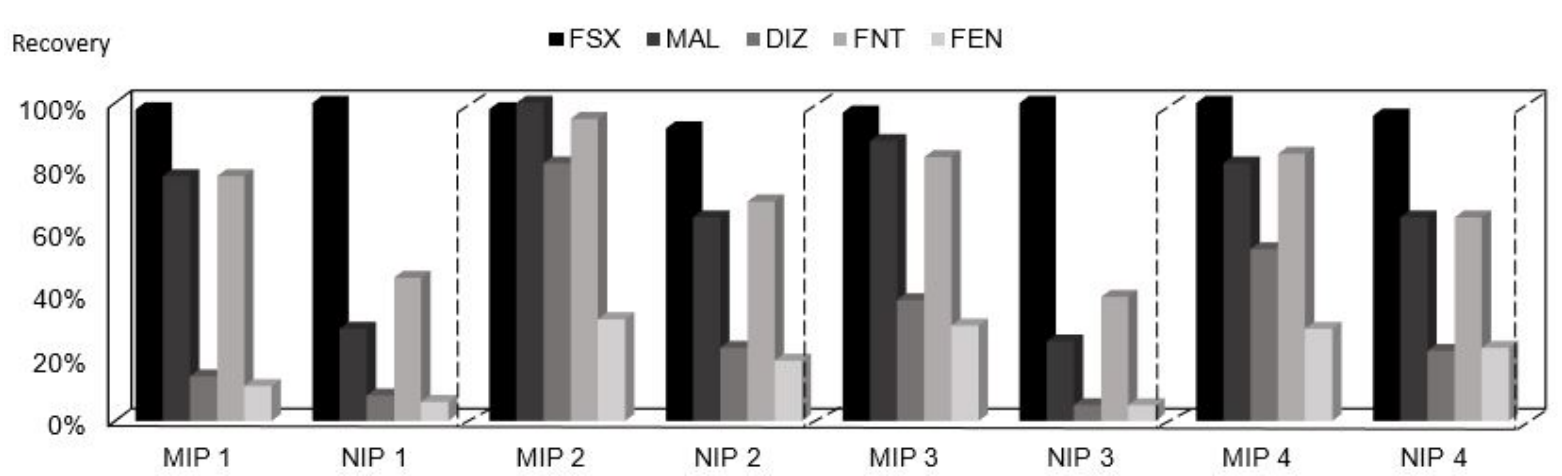

Figure 2: Recovery of five OPs in the elution fraction obtained on four MIPs/NIPs by applying the screening extraction procedure including the percolation of $1 \mathrm{~mL}$ of hexane spiked with $1 \mathrm{mg} / \mathrm{L}$ of each OP, a washing with $1 \mathrm{~mL}$ of hexane/DCM 95/5 (v/v) and an elution with $1 \mathrm{~mL}$ of ACN. The average recovery $(\%) \pm S D(n=3)$ for MIP/NIP 2 and MIP/NIP 4 and the average recovery $(\%),(n=2)$ for MIP 1 and MIP 3 are reported.

The comparison of MIPs and NIPs in these conditions shows that no selectivity (MIP 1 and MIP 3 ) or a low selectivity (MIP 2 and MIP 4) was obtained for FSX, which is the most polar of the studied compound. At this stage, an improvement in selectivity for all the MIPs could be expected for this strongly retained compound by increasing the elution strength of the washing. Nevertheless, MIP 4 presented a good retention for the five OPs but a lower selectivity than the three other MIPs for MAL and FEN. MIP 4 was then removed from the study. The three other MIPs were very similar in terms of retention and selectivity. However, MIP 1 appears less retentive than MIPs 2 and 3 (especially for DIZ and FEN). Finally, according to the retention and the selectivity observed for DIZ that was higher on MIP 2 than on MIP 3, MIP 2 was selected and named MIP for the rest of the study.

Additional studies were carried out on this MIP to improve the retention of these four compounds by changing the elution strength of the washing solution using $7 \%$ or $3 \%$ of DCM instead of $5 \%$, but no improvement was observed in terms of selectivity or retention.

\subsection{Potential of the MIPs towards OPs}

To evaluate the potential of this MIP for the selective extraction of the ten OPs, the previously developed extraction procedure was further applied in triplicate to the ten OPs of interest (Figure 3A). To confirm its potential, the same experiment was carried out on a MIP resulting from a second independent synthesis (Figure 3B). The extraction profile represents recovery obtained in the percolation, washing and elution fractions on MIP and on NIP (Figure $3 \mathrm{~A}$ and $3 \mathrm{~B}$ ). The target OPs can be gathered together in three different groups according to their behavior on MIP/NIP. The MIP does not present any selectivity for the most polar OPs (DIM, FSX, FSN and DMT) because the retention was strong (up to the elution fraction) and was the same on MIP and NIP. Some selectivity was obtained for the non-polar OPs (PIM and CLE) because their extraction profiles on MIP and NIP were different but their retention was low since they were mainly recovered in the washing fraction. In return, a high retention and a satisfactory selectivity for moderately polar compounds was obtained. For example, the recovery of DIZ in the elution fraction was $81 \pm 8 \%$ for the MIP and $23 \pm 11 \%$ for the NIP. SD values between 2 and $13 \%(n=3)$ also indicate the good repeatability of this MIP-SPE procedure. 


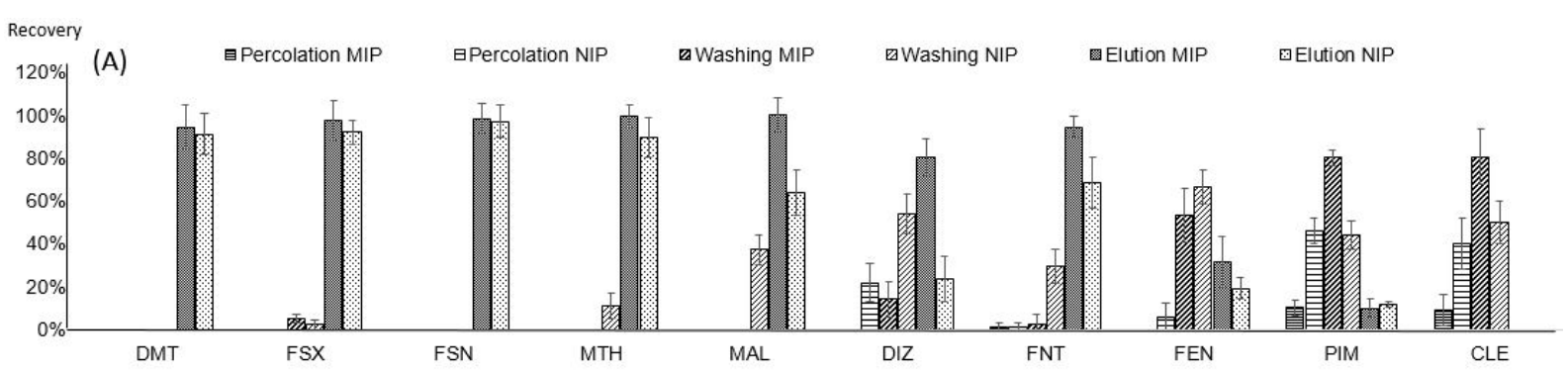

(B)

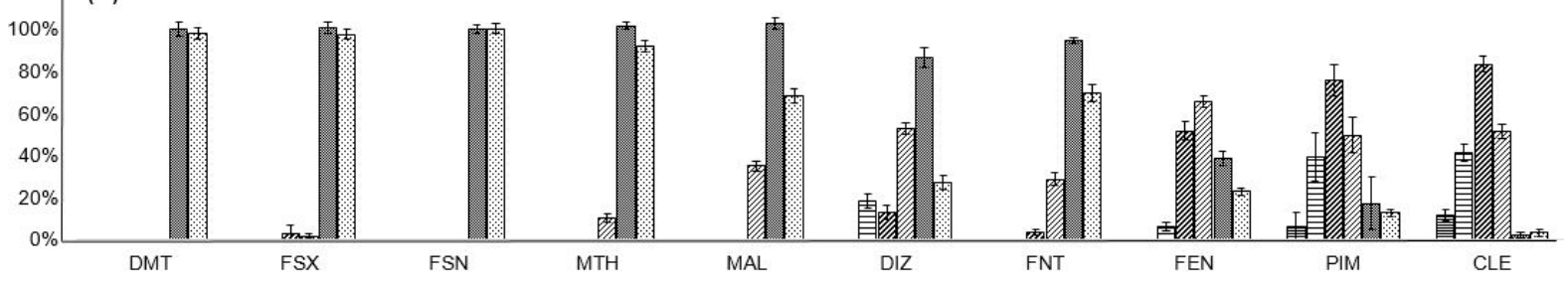

Figure 3: Extraction profiles obtained when percolating the ten OPs (A) on MIP/NIP ( $n=3$ assays) and (B) on two MIPs/NIPs synthesized independently using the same condition of synthesis ( $n=3$ assays on each synthesis of MIP, $n=6$ ). The extraction procedure was the same as in Figure 2.

In addition, the extraction profiles obtained for the two syntheses were similar as demonstrated by results reported on Figure 3B that corresponds to the average extraction profiles observed with the use of the MIPs/NIPs resulting from two different syntheses (extraction in triplicates on both MIP/NIP, $n=6$ ). Indeed SD values of the recovery were between 3 and 12\%. Moreover, the analysis of variance, ANOVA test, $(\alpha=5 \%)$ demonstrated that there was not a significant variation between recoveries obtained on the MIPs resulting from the two syntheses. These last two observations show the good repeatability of the extraction procedure and the reliability of the synthesis.

\subsection{Study of the capacity of the MIP in pure media}

The capacity of the MIP, which corresponds to the maximum amount of a compound that can be retained by the imprinted polymer with a constant recovery, was studied. This parameter is linked to the number of specific cavities that are available for the trapping of the target compounds. Therefore, the determination of the capacity was performed using MAL, which presents a selective behaviour on this polymer as shown in pure media (Figure 3A): recoveries of $100 \%$ with a SD of $8 \%$ on the MIP and of $64 \%$ with a SD of $11 \%$ on the NIP. To determine this capacity, samples of hexane were spiked with increasing amounts of MAL and percolated on MIP and on NIP and the extraction procedure described on Figure 3 was applied to each sample. The amounts of MAL in the elution fraction of the MIP were plotted as a function of the percolated amounts. The resulting curve reported on Figure 4 presents two different parts. For the lowest percolated amounts of MAL, the trend is linear, meaning that there is a constant recovery of extraction for this range of percolated amounts. The slope of this linear part corresponds to a recovery of $113 \%$. This value was very close to the recoveries previously obtained for MAL using the same extraction procedure (Figure 3A). For higher amounts of MAL loaded on the MIP, the curve reaches a plateau. The recovery decreases since the capacity of the cartridge was overloaded. Considering the point where the two parts of the curve intercept as the maximum amount of MAL retained on the MIP with constant recovery, the capacity can be estimated at about $32 \mu \mathrm{g}$ of MAL for $32 \mathrm{mg}$ of MIP, which corresponds to a capacity of about $1 \mathrm{mg} / \mathrm{g}$ or to $3.31 \mu \mathrm{mol} / \mathrm{g}$ of MIP. Over this value, 
quantitative analyses are not reliable since there is a decrease in the recovery of extraction. This capacity value was in good agreement with the capacity values reported in the literature namely, between $0.37 \mu \mathrm{mol} / \mathrm{g}$ and $40 \mu \mathrm{mol} / \mathrm{g}[17,42,43]$.

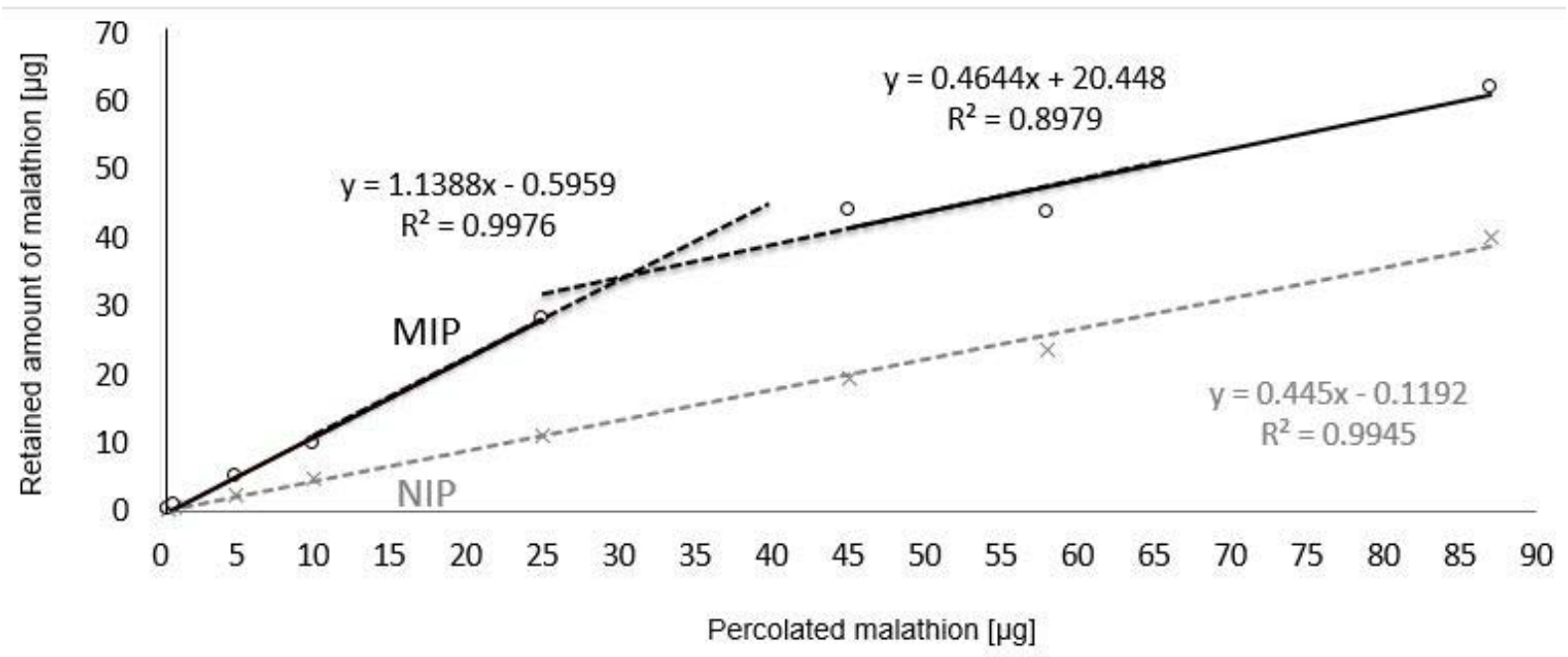

Figure 4: Calibration curves obtained by plotting the amount of malathion recovered in the elution fraction of the MIP and the corresponding NIP after the percolation of different amounts of malathion spiked in $1 \mathrm{~mL}$ hexane. The extraction procedure was the same as in Figure 2.

\subsection{Extraction of OPs from different oils}

\subsubsection{Preliminary study of the repeatability of the extraction procedure in different vegetable oils}

In order to evaluate the potential of the MIP for the extraction of OPs from real media, three vegetable oils (sunflower, almond and olive oils) were spiked at $2.5 \mathrm{mg} / \mathrm{kg}$ with the nine OPs. The analysis was carried out with LC-UV for FNT and with LC-MS for the other OPs. Despite the selectivity obtained for FEN in pure media, it was not considered in this study because its LOQ in MS or in UV were too high for its determination at this spiking level in the fractions resulting from SPE on MIP. Figure 5 shows the recoveries of the nine OPs in the elution fraction for the three oils. Recoveries obtained in pure media were also reported on this figure. The results obtained for oil samples confirmed the results on pure media: the MIP was not selective towards the most polar OPs (DMT, FSX and FSN) with this extraction procedure. Moreover, a matrix effect causes a decreased of recovery for all the compounds, this recovery being lower for spiked oil samples than for spiked pure media. Therefore, the less polar compounds that were only slightly retained in pure media were weakly or no more retained on the MIP/NIP.

For the moderately polar OPs, MTH, MAL, DIZ and FNT, the retention was lower (especially for DIZ) than in pure media because of the matrix effect. However, the selectivity of the extraction on MIP was maintained, even slightly improved as shown by the highest difference between recoveries on MIP and NIP. Indeed, MIP becomes more selective towards MTH in oil samples than in pure media. This can be explained by the fact that the matrix components may greatly weaken the non-specific interactions as compared with the specific ones. To improve the recoveries for these OPs, the optimization of the SPE procedure appears to be necessary. 


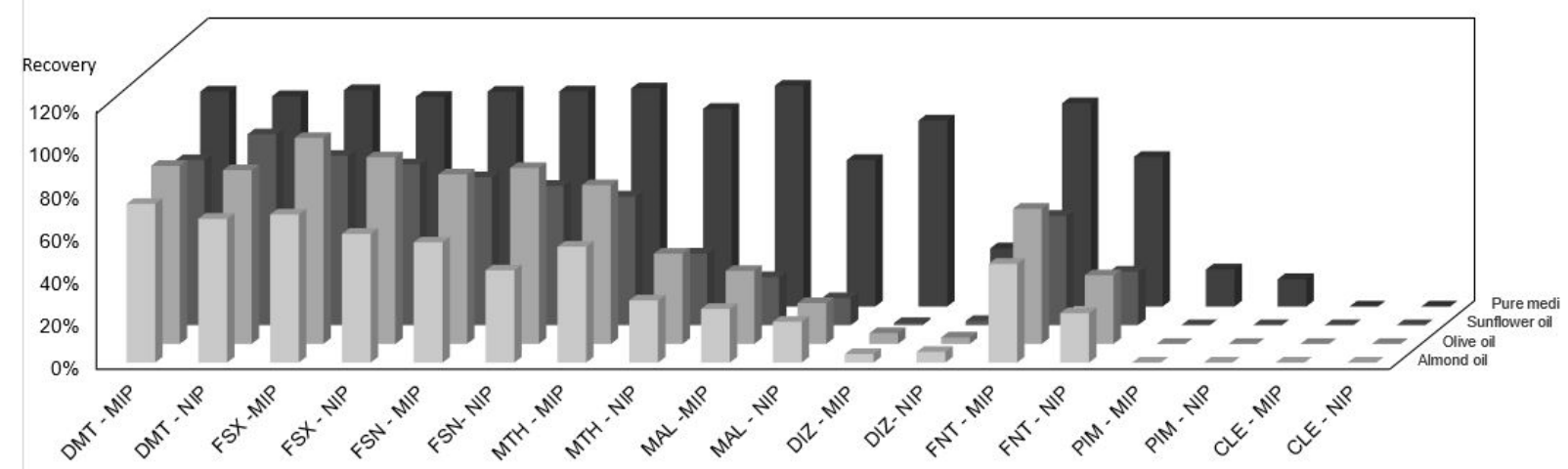

Figure 5: Recovery obtained on MIP/NIP after applying the extraction procedure on different types of vegetable oils (almond, olive and sunflower) spiked at $2.5 \mathrm{mg} / \mathrm{kg}$ with nine OPs. Extraction conditions: see part 2.5.1. Recovery obtained in pure medium (spiked hexane) correspond to those already reported in Figure $3 \mathrm{~A}$.

\subsubsection{Optimization of the SPE procedure using almond oil}

As the extraction profile (Figure 5) seems not to be affected by the nature of the oil, being similar for the three types of oils, this optimization was only carried out for almond oil samples. Despite the selectivity obtained for the four moderately polar compounds, this part of the study only focusses on the three OPs that can be analyzed by LC-MS. The MRLs values were taken as reference to set the spiking level of OPs in the almond oil. Nevertheless, the MRLs for pesticides in processed products like crude oils (and refined oils) are not specifically set in the EU legislation. To compare the LOQs of pesticides in crude oils with the MRLs of pesticides in seeds, a processing factor proposed by FIEDOL (the vegetable oil and protein meal industry association) was applied. This processing factor is calculated taking a count the oil content and the hydrophobicity of the OPs at the same time. As an example, the proposed processing factor for hydrophobic pesticides $(\log \mathrm{P}=3)$ in nut seed with $54 \%$ in oil content was 2.5. For this case, the average oil content of almond oil is 58\% [44], thus the estimate processing factor for this oil was 2.6. This proposal value, was used to estimate the LOQs of OPs in almond seed (see Table 3). The almond extract was spiked at $100 \mu \mathrm{g} / \mathrm{kg}$ oil, instead of $2.5 \mathrm{mg} / \mathrm{kg}$, and diluted 10 times to limit the matrix effect during the extraction procedure on MIP/NIP as proposed by Barkas et al. for olive oil [17]. In addition, different volumes of washing solution hexane and DCM $(95 / 5, \mathrm{v} / \mathrm{v})$ were tested: $0.4,0.65,0.8$ and $1 \mathrm{~mL}$.

The washing with $0.65 \mathrm{~mL}$ presented a good compromise in terms of recovery and selectivity. Indeed, for 0.8 and $1 \mathrm{~mL}$ the extraction recovery decreases particularly for MAL and DIZ. In return, if a washing with $0.4 \mathrm{~mL}$ gives rise to the same recoveries as for $0.65 \mathrm{~mL}$, this higher volume was preferred because it must allow the removal of a higher amount of matrix components than the smaller one. Recoveries in the elution fraction obtained by applying the dilution of the extract and this washing volume to almond oil extract are reported in Table 3. Recoveries of extraction were corrected for MAL by taking into account the low amount of this compound $(4 \mu \mathrm{g} / \mathrm{kg}$ ) detected in the blank oil sample. The SD values were between 4 and $6 \%(n=3)$. Those values are comparable to those obtained in pure media (between 5 and $8 \%$ ). The selectivity is highlighted by the higher recoveries obtained on MIP (between 73 and 99\%) than on NIP (between 34 and 75\%).

Table 3: Recovery obtained in the elution fraction using almond oil spiked with $100 \mu \mathrm{g} / \mathrm{kg}$ of the three OPs after LLE and SPE clean-up using MIP/NIP or C18. LOQs correspond to $\mathrm{S} / \mathrm{N}=10{ }^{\mathrm{a}}$ : MRLs according 
to EU regulation $\mathrm{N}^{\circ} 396 / 2005 ;{ }^{\text {b}}$ : processing factor from FEDIOL (vegetable oil and protein meal industry association); ${ }^{c}$ : estimated LOQs according to FEDIOL processing factor.

\begin{tabular}{|c|c|c|c|c|c|c|c|}
\hline OPs & $\begin{array}{l}\text { MRLs in } \\
\text { almond } \\
\text { seed } \\
(\mu \mathrm{g} / \mathrm{kg})\end{array}$ & Sorbent & $\begin{array}{c}\text { Recovery } \\
\text { (\%) }\end{array}$ & $\begin{array}{l}\text { LOQ in } \\
\text { almond } \\
\text { oil }(\mu \mathrm{g} / \mathrm{kg})\end{array}$ & $\begin{array}{c}\text { Processing } \\
\text { factor }^{b}\end{array}$ & $\begin{array}{c}\text { Estimated } \\
\text { LOQs }{ }^{c} \text { in } \\
\text { almond seed } \\
(\mu \mathrm{g} / \mathrm{kg})\end{array}$ & $\begin{array}{c}\text { Matrix } \\
\text { effect } \\
(\%)\end{array}$ \\
\hline & & MIP & $99 \pm 6$ & $2 \pm 1$ & 2.6 & $0.8 \pm 0.4$ & $7 \pm 3$ \\
\hline \multirow[t]{3}{*}{ MTH } & 50 & NIP & $75 \pm 13$ & & & & \\
\hline & & C18 & $106 \pm 1$ & & & & $21 \pm 6$ \\
\hline & & MIP & $73 \pm 4$ & $5 \pm 1$ & 2.6 & $2 \pm 0.4$ & $17 \pm 8$ \\
\hline \multirow[t]{3}{*}{ MAL } & 20 & NIP & $42 \pm 5$ & & & & \\
\hline & & C18 & $115 \pm 7$ & & & & $34 \pm 13$ \\
\hline & & MIP & $81 \pm 6$ & $0.8 \pm 0.3$ & 2.6 & $0.3 \pm 0.1$ & $11 \pm 3$ \\
\hline \multirow[t]{2}{*}{$\mathrm{DIZ}$} & 50 & NIP & $34 \pm 8$ & & & & \\
\hline & & $\mathrm{C} 18$ & $134 \pm 9$ & & & & $35 \pm 8$ \\
\hline
\end{tabular}

These results were also compared with those obtained by using conventional C18 silica sorbents after the same LLE step (Table 3). For this, the same spiked level was used for the three OPs in almond oil as the objective was also to compare the matrix effect in similar conditions. The recovery for the three OPs was over $100 \%$ using $\mathrm{C} 18$ silica, which indicates that the results obtained using $\mathrm{C} 18$ silica could be affected by a matrix effect. The contribution of matrix effect in the quantification of OPs was then evaluated for both sorbents. After applying the whole extraction procedure to a non-spiked oil sample (LLE and SPE on MIP or on C18), the elution fraction was spiked with three OPs. This extract was injected in LC-MS, and the obtained signals were compared to those of a standard solution in pure media at same concentration level. The results indicate that the contribution of matrix effects using C18 silica was higher than when using the MIP. As an example, for MTH, the matrix effect was $21 \%$ on $\mathrm{C} 18$ and $7 \%$ on MIP. Therefore, the comparison of the two extraction procedures performed on MIP and C18 indicates that the use of MIP as a selective sorbent limits the matrix effect that occurs when using a conventional sorbent by a factor two to three. This can be explained by a more efficient removal of matrix components from the MIP than from C18 silica. This can also be illustrated by comparing the LC-UV $(210 \mathrm{~nm})$ chromatograms resulting from the analysis of the elution fraction after using C18 or MIP (Figure 6). The chromatogram corresponding to $\mathrm{C} 18$ contains more peaks of interfering compounds than those obtained from MIPs. The MIP allowing a larger part of interfering compounds to being removed thus improving the reliability of LC-UV and LC-MS analyses.

Figure S2 (supplementary material 2) corresponds to the LC-MS analysis in (MRM mode) of the elution fraction from the MIP for an almond oil sample spiked at $100 \mu \mathrm{g} / \mathrm{kg}$. The calculated LOQs for the three target OPs (MTH, MAL and DIZ) are reported in Table 3 and range from 0.3 to $2 \mu \mathrm{g} / \mathrm{kg}$ in almond seed. These results mean that this analytical method allows the determination of concentration levels of OPs lower than their MRLs (20 to 50 $\mu \mathrm{g} / \mathrm{kg}$ ). Moreover, it is important to mention than these results obtained for vegetable oils (comparison of oils, optimization of washing conditions, studies of matrix effect) were achieved on the same MIP without observing any decrease in its performance, thus highlighting its good chemical stability and its reusability for more than 100 experiments. 


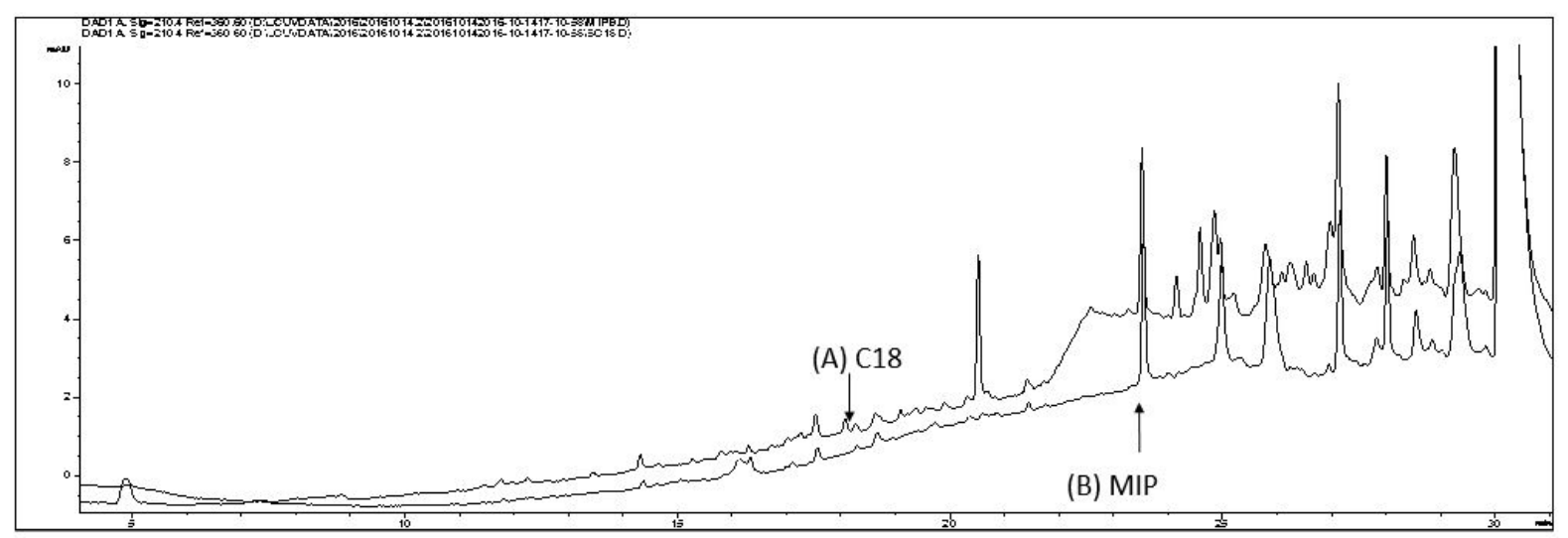

Figure 6: LC-UV chromatograms $(210 \mathrm{~nm})$ of elution fraction of almond oil extract spiked at $100 \mu \mathrm{g} / \mathrm{kg}$ with eight OPs using C18 or MIP after LLE. The extraction procedure was described in the part 2.5.2 (washing volume of $0.65 \mathrm{~mL}$ ).

\section{Conclusions}

Different conditions of synthesis were screened to determine those that allow the synthesis of a MIP able to selectively extract OPs that belong to a very broad range of molecular structures and log $P$ values (between 0.7 and 4.7). Among the six synthesized MIPs, one of them was able to selectively trap five OPs (MTH, MAL, DIZ, FNT and FEN).

After studying the repeatability of the optimized SPE procedure and of the reliability of the MIP synthesis in pure media, the performances of this polymer were evaluated in real media. The retention of OPs on the MIP was similar using three different oils (olive, sunflower and almond oils). Therefore, a rapid optimization of the SPE procedure on almond oil was achieved and allowed us to obtain recoveries for three OPs (MTH, MAL and DIZ) between 73 and $99 \%$ using the MIP and of only 34 to $75 \%$ using the NIP. The MIP also allows the matrix effects to being reduced by a factor of two to three: the matrix effects were between 7 and $11 \%$ using the MIP and between 21 and 35\% using the C18 silica sorbent for a sample of almond oil spiked at $100 \mu \mathrm{g} / \mathrm{kg}$. The LOQs obtained for almond seeds (between 0.3 and 2 $\mu \mathrm{g} / \mathrm{kg}$, estimated taking a count the LOQs of spiked almond oil), were lower than the MRLs (between 20 and $50 \mu \mathrm{g} / \mathrm{kg}$ ) established for the almond seeds.

\section{References}

[1] A.G. Sánchez, N.R. Martos, E. Ballesteros, Multiresidue analysis of pesticides in olive oil by gel permeation chromatography followed by gas chromatography-tandem massspectrometric determination, Anal. Chim. Acta. 558 (2006) 53-61. doi:10.1016/j.aca.2005.11.019.

[2] L. Li, Z. Zhou, C. Pan, C. Qian, S. Jiang, F. Liu, Determination of Organophosphorus Pesticides in Soybean Oil, Peanut Oil and Sesame Oil by Low-Temperature Extraction and GC-FPD, Chromatographia. 66 (2007) 625-629. doi:10.1365/s10337-007-0349-9.

[3] L. Polgar, B. Kmellar, J.F. Garcia-Reyes, P. Fodor, Comprehensive evaluation of the cleanup step in QuEChERS procedure for the multi-residue determination of pesticides in different vegetable oils using LC-MS/MS, Anal. Methods. 4 (2012) 1142-1148. doi:10.1039/C2AY05543K.

[4] S. Chawla, H.K. Patel, K.M. Vaghela, F.K. Pathan, H.N. Gor, A.R. Patel, P.G. Shah, Development and validation of multiresidue analytical method in cotton and groundnut 
oil for 87 pesticides using low temperature and dispersive cleanup on gas chromatography and liquid chromatography-tandem mass spectrometry, Anal. Bioanal. Chem. 408 (2016) 983-997. doi:10.1007/s00216-015-9192-2.

[5] Z. He, Y. Wang, L. Wang, Y. Peng, W. Wang, X. Liu, Determination of 255 pesticides in edible vegetable oils using QuEChERS method and gas chromatography tandem mass spectrometry, Anal. Bioanal. Chem. 409 (2017) 1017-1030. doi:10.1007/s00216-0160016-9.

[6] L. Chen, X. Wang, W. Lu, X. Wu, J. Li, Molecular imprinting: perspectives and applications, Chem. Soc. Rev. 45 (2016) 2137-2211. doi:10.1039/C6CS00061D.

[7] R. Schirhagl, Bioapplications for Molecularly Imprinted Polymers, Anal. Chem. 86 (2014) 250-261. doi:10.1021/ac401251j.

[8] V. Pichon, Selective sample treatment using molecularly imprinted polymers, J. Chromatogr. A. 1152 (2007) 41-53.

[9] C. Algieri, E. Drioli, L. Guzzo, L. Donato, Bio-Mimetic Sensors Based on Molecularly Imprinted Membranes, Sensors. 14 (2014) 13863-13912.

[10] B. Sellergren, Direct Drug Determination by Selective Sample Enrichment on an Imprinted Polymer, Anal. Chem. 66 (1994) 1578-1582. doi:10.1021/ac00081a036.

[11] X. Zhu, J. Yang, Q. Su, J. Cai, Y. Gao, Selective solid-phase extraction using molecularly imprinted polymer for the analysis of polar organophosphorus pesticides in water and soil samples, J. Chromatogr. A. 1092 (2005) 161-169. doi:10.1016/j.chroma.2005.07.037.

[12] X. Zhu, Q. Su, J. Cai, J. Yang, Y. Gao, Molecularly imprinted polymer membranes for substance-selective solid-phase extraction from aqueous solutions, J. Appl. Polym. Sci. 101 (2006) 4468-4473. doi:10.1002/app.24183.

[13] L.A. Pereira, S. Rath, Molecularly imprinted solid-phase extraction for the determination of fenitrothion in tomatoes, Anal. Bioanal. Chem. 393 (2008) 1063-1072. doi:10.1007/s00216-008-2511-0.

[14] Z.-L. Shen, X.-L. Zhu, J. Yang, J.-B. Cai, Q.-D. Su, Study on the Binding Characteristic of Methamidophos-Specific Molecularly Imprinted Polymer and the Interactions between Template and Monomers, J. Chin. Chem. Soc. 55 (2008) 587-593. doi:10.1002/jccs.200800086.

[15] Z. Xu, G. Fang, S. Wang, Molecularly imprinted solid phase extraction coupled to highperformance liquid chromatography for determination of trace dichlorvos residues in vegetables, Food Chem. 119 (2010) 845-850. doi:10.1016/j.foodchem.2009.08.047.

[16] Shen, Zhong-Lan, D. Yuan, Q.-D. SU, H. Zhang, J. Wang, J.-H. Zhu, Y.-M. Liu, Selective Solid-Phase Extraction Using Molecularly Imprinted Polymer for Analysis of Methamidophos in Water and Soil Samples, Biosci. Biotechnol. Biochem. 75 (2011) 473479. doi:10.1271/bbb.100668.

[17] I. Bakas, N. Ben Oujji, E. Moczko, G. Istamboulie, S. Piletsky, E. Piletska, I. Ait-Ichou, E. Ait-Addi, T. Noguer, R. Rouillon, Molecular imprinting solid phase extraction for selective detection of methidathion in olive oil, Anal. Chim. Acta. 734 (2012) 99-105. doi:10.1016/j.aca.2012.05.013.

[18] J. Xin, X. Qiao, Y. Ma, Z. Xu, Simultaneous separation and determination of eight organophosphorous pesticide residues in vegetables through molecularly imprinted solid-phase extraction coupled to gas chromatography, J. Sep. Sci. 35 (2012) 3501-3508. doi:10.1002/jssc.201200754.

[19] I. Bakas, N.B. Oujji, E. Moczko, G. Istamboulie, S. Piletsky, E. Piletska, E. Ait-Addi, I. Ait-Ichou, T. Noguer, R. Rouillon, Computational and experimental investigation of 
molecular imprinted polymers for selective extraction of dimethoate and its metabolite omethoate from olive oil, J. Chromatogr. A. 1274 (2013) 13-18.

doi:10.1016/j.chroma.2012.11.061.

[20] J. Xin, X. Qiao, Z. Xu, J. Zhou, Molecularly Imprinted Polymer as Sorbent for SolidPhase Extraction Coupling to Gas Chromatography for the Simultaneous Determination of Trichlorfon and Monocrotophos Residues in Vegetables, Food Anal. Methods. 6 (2013) 274-281. doi:10.1007/s12161-012-9432-4.

[21] M.M. Sanagi, S. Salleh, W.A.W. Ibrahim, A.A. Naim, D. Hermawan, M. Miskam, I. Hussain, H.Y. Aboul-Enein, Molecularly imprinted polymer solid-phase extraction for the analysis of organophosphorus pesticides in fruit samples, J. Food Compos. Anal. 32 (2013) 155-161. doi:10.1016/j.jfca.2013.09.001.

[22] Q. Wang, X. Zhang, Z. Xu, H. Gao, Simultaneous Determination of Three Trace Organophosphorus Pesticide Residues in Vegetables Using Molecularly Imprinted SolidPhase Extraction Coupled with High-Performance Liquid Chromatography, Food Anal. Methods. 8 (2015) 2044-2051. doi:10.1007/s12161-014-0086-2.

[23] I. Bakas, N. Ben Oujji, G. Istamboulié, S. Piletsky, E. Piletska, E. Ait-Addi, I. Ait-Ichou, T. Noguer, R. Rouillon, Molecularly imprinted polymer cartridges coupled to high performance liquid chromatography (HPLC-UV) for simple and rapid analysis of fenthion in olive oil, Talanta. 125 (2014) 313-318. doi:10.1016/j.talanta.2014.03.020.

[24] D. Davoodi, M. Hassanzadeh-Khayyat, M. Asgharian Rezaei, S.A. Mohajeri, Preparation, evaluation and application of diazinon imprinted polymers as the sorbent in molecularly imprinted solid-phase extraction and liquid chromatography analysis in cucumber and aqueous samples, Food Chem. 158 (2014) 421-428. doi:10.1016/j.foodchem.2014.02.144.

[25] H.G. Zuo, J.X. Zhu, C.R. Zhan, L. Shi, M. Xing, P. Guo, Y. Ding, H. Yang, Preparation of malathion MIP-SPE and its application in environmental analysis, Environ. Monit. Assess. 187 (2015) 1-19. doi:10.1007/s10661-015-4641-0.

[26] J. He, L. Song, S. Chen, Y. Li, H. Wei, D. Zhao, K. Gu, S. Zhang, Novel restricted access materials combined to molecularly imprinted polymers for selective solid-phase extraction of organophosphorus pesticides from honey, Food Chem. 187 (2015) 331337. doi:10.1016/j.foodchem.2015.04.069.

[27] Q. Lu, X. Chen, L. Nie, J. Luo, H. Jiang, L. Chen, Q. Hu, S. Du, Z. Zhang, Tuning of the vinyl groups' spacing at surface of modified silica in preparation of high density imprinted layer-coated silica nanoparticles: A dispersive solid-phase extraction materials for chlorpyrifos, Talanta. 81 (2010) 959-966. doi:10.1016/j.talanta.2010.01.044.

[28] S. Xu, C. Guo, Y. Li, Z. Yu, C. Wei, Y. Tang, Methyl parathion imprinted polymer nanoshell coated on the magnetic nanocore for selective recognition and fast adsorption and separation in soils, J. Hazard. Mater. 264 (2014) 34-41. doi:10.1016/j.jhazmat.2013.10.060.

[29] F. Zare, M. Ghaedi, A. Daneshfar, A. Ostovan, Magnetic molecularly imprinted polymer for the efficient and selective preconcentration of diazinon before its determination by high-performance liquid chromatography, J. Sep. Sci. 38 (2015) 27972803.

[30] J.-J. Du, R.-X. Gao, H. Yu, X.-J. Li, H. Mu, Selective extraction of dimethoate from cucumber samples by use of molecularly imprinted microspheres, J. Pharm. Anal. 5 (2015) 200-206. doi:10.1016/j.jpha.2014.10.004. 
[31] X. Luo, C. Li, Y. Duan, H. Zhang, D. Zhang, C. Zhang, G. Sun, X. Sun, Molecularly imprinted polymer prepared by Pickering emulsion polymerization for removal of acephate residues from contaminated waters, J. Appl. Polym. Sci. 133 (2016) 43126. doi:10.1002/app.43126.

[32] X. Wang, X. Qiao, Y. Ma, T. Zhao, Z. Xu, Simultaneous Determination of Nine Trace Organophosphorous Pesticide Residues in Fruit Samples Using Molecularly Imprinted Matrix Solid-Phase Dispersion Followed by Gas Chromatography, J. Agric. Food Chem. 61 (2013) 3821-3827. doi:10.1021/jf400269q.

[33] M. Zhou, N. Hu, S. Shu, M. Wang, Molecularly Imprinted Nanomicrospheres as Matrix Solid-Phase Dispersant Combined with Gas Chromatography for Determination of Four Phosphorothioate Pesticides in Carrot and Yacon, J. Anal. Methods Chem. 2015 (2015) 11 pages. doi:10.1155/2015/385167.

[34] F.X. Liang, X.L. Zhu, J. Yang, Q.D. Su, Accelerated solvent extraction and matrix solid phase dispersion using molecularly imprinted polymer for the analysis of monocrotophos in soil, Asian J. Chem. 20 (2008) 3954-3960.

[35] H. Hashemi-Moghaddam, D.J. Jedi, Solid-phase microextraction of chlorpyrifos in fruit samples by synthesised monolithic molecularly imprinted polymer fibres, Int. J. Environ. Anal. Chem. 95 (2015) 33-44. doi:10.1080/03067319.2014.983495.

[36] Y.-L. Wang, Y.-L. Gao, P.-P. Wang, H. Shang, S.-Y. Pan, X.-J. Li, Sol-gel molecularly imprinted polymer for selective solid phase microextraction of organophosphorous pesticides, Talanta. 115 (2013) 920-927. doi:10.1016/j.talanta.2013.06.056.

[37] J.-W. Li, Y.-L. Wang, S. Yan, X.-J. Li, S.-Y. Pan, Molecularly imprinted calixarene fiber for solid-phase microextraction of four organophosphorous pesticides in fruits, Food Chem. 192 (2016) 260-267. doi:10.1016/j.foodchem.2015.07.018.

[38] X. Zhu, J. Cai, J. Yang, Q. Su, Y. Gao, Films coated with molecular imprinted polymers for the selective stir bar sorption extraction of monocrotophos, J. Chromatogr. A. 1131 (2006) 37-44. doi:10.1016/j.chroma.2006.07.041.

[39] X. Wang, Q. Tang, Q. Wang, X. Qiao, Z. Xu, Study of a molecularly imprinted solidphase extraction coupled with high-performance liquid chromatography for simultaneous determination of trace trichlorfon and monocrotophos residues in vegetables, J. Sci. Food Agric. 94 (2014) 1409-1415. doi:10.1002/jsfa.6429.

[40] C. Alexander, H.S. Andersson, L.I. Andersson, R.J. Ansell, N. Kirsch, I.A. Nicholls, J. O'Mahony, M.J. Whitcombe, Molecular imprinting science and technology: a survey of the literature for the years up to and including 2003, J. Mol. Recognit. JMR. 19 (2006) 106-180. doi:10.1002/jmr.760.

[41] V. Pichon, Selective sample treatment using molecularly imprinted polymers, J. Chromatogr. A. 1152 (2007) 41-53. doi:10.1016/j.chroma.2007.02.109.

[42] V. Thibert, P. Legeay, F. Chapuis-Hugon, V. Pichon, Synthesis and characterization of molecularly imprinted polymers for the selective extraction of cocaine and its metabolite benzoylecgonine from hair extract before LC-MS analysis, Talanta. 88 (2012) 412-419. doi:10.1016/j.talanta.2011.11.009.

[43] P. Svoboda, A. Combes, J. Petit, L. Nováková, V. Pichon, B. group, Synthesis of a molecularly imprinted sorbent for selective solid-phase extraction of $\beta-\mathrm{N}$-methylamino-Ialanine, Talanta. 144 (2015) 1021-1029. doi:10.1016/j.talanta.2015.07.052.

[44] A.N. Yildirim, F. Akinci-Yildirim, B. Şan, Y. Sesli, Total Oil Content and Fatty Acid Profile of some Almond (Amygdalus Communis L.) Cultivars, Pol. J. Food Nutr. Sci. 66 (2016) 173-178. doi:10.1515/pjfns-2015-0032. 


\section{Supplementary material 1}

\begin{tabular}{|c|c|c|c|c|c|c|}
\hline OPs & $\begin{array}{l}\text { Precursor Ion } \\
\text { m/z (Da) }\end{array}$ & $\begin{array}{l}\text { Tube } \\
\text { lens } \\
\text { (V) }\end{array}$ & $\begin{array}{l}\text { Quantitation } \\
\text { ion } \mathrm{m} / \mathrm{z} \\
\text { (Da) }\end{array}$ & $\begin{array}{l}\text { Collision energy of } \\
\text { quantitation ion } \\
\text { (V) }\end{array}$ & $\begin{array}{c}\text { Confirming } \\
\text { Ion } \mathrm{m} / \mathrm{z} \\
\text { (Da) }\end{array}$ & $\begin{array}{c}\mathbf{t}_{\mathrm{R}} \\
(\mathrm{min}) \\
\end{array}$ \\
\hline DMT & {$[\mathrm{M}+\mathrm{H}]^{+}=230$} & 90 & 125 & 22 & 170 & 7.7 \\
\hline FSX & {$[\mathrm{M}+\mathrm{H}]^{+}=295$} & 116 & 280 & 18 & 109 & 17.7 \\
\hline FSN & {$\left[\mathrm{M}+\mathrm{NH}_{4}\right]^{+}=328$} & 85 & 311 & 12 & 125 & 19.0 \\
\hline MTH & {$\left[\mathrm{M}+\mathrm{NH}_{4}\right]^{+}=320$} & 60 & 145 & 13 & 85 & 19.5 \\
\hline MAL & {$\left[\mathrm{M}+\mathrm{NH}_{4}\right]^{+}=348$} & 81 & 127 & 17 & 99 & 22.12 \\
\hline $\mathrm{DIZ}$ & {$[\mathrm{M}+\mathrm{H}]^{+}=305$} & 96 & 169 & 21 & 153 & 23.9 \\
\hline PIM & {$[\mathrm{M}+\mathrm{H}]^{+}=306$} & 96 & 164 & 22 & 108 & 24.8 \\
\hline CLE & {$[\mathrm{M}+\mathrm{H}]^{+}=352$} & 112 & 200 & 21 & 97 & 26.3 \\
\hline
\end{tabular}

Table S1. Tube lens values corresponding to quantitation and confirming ions and collision energies corresponding to quantitation ions.

\section{Supplementary material 2}

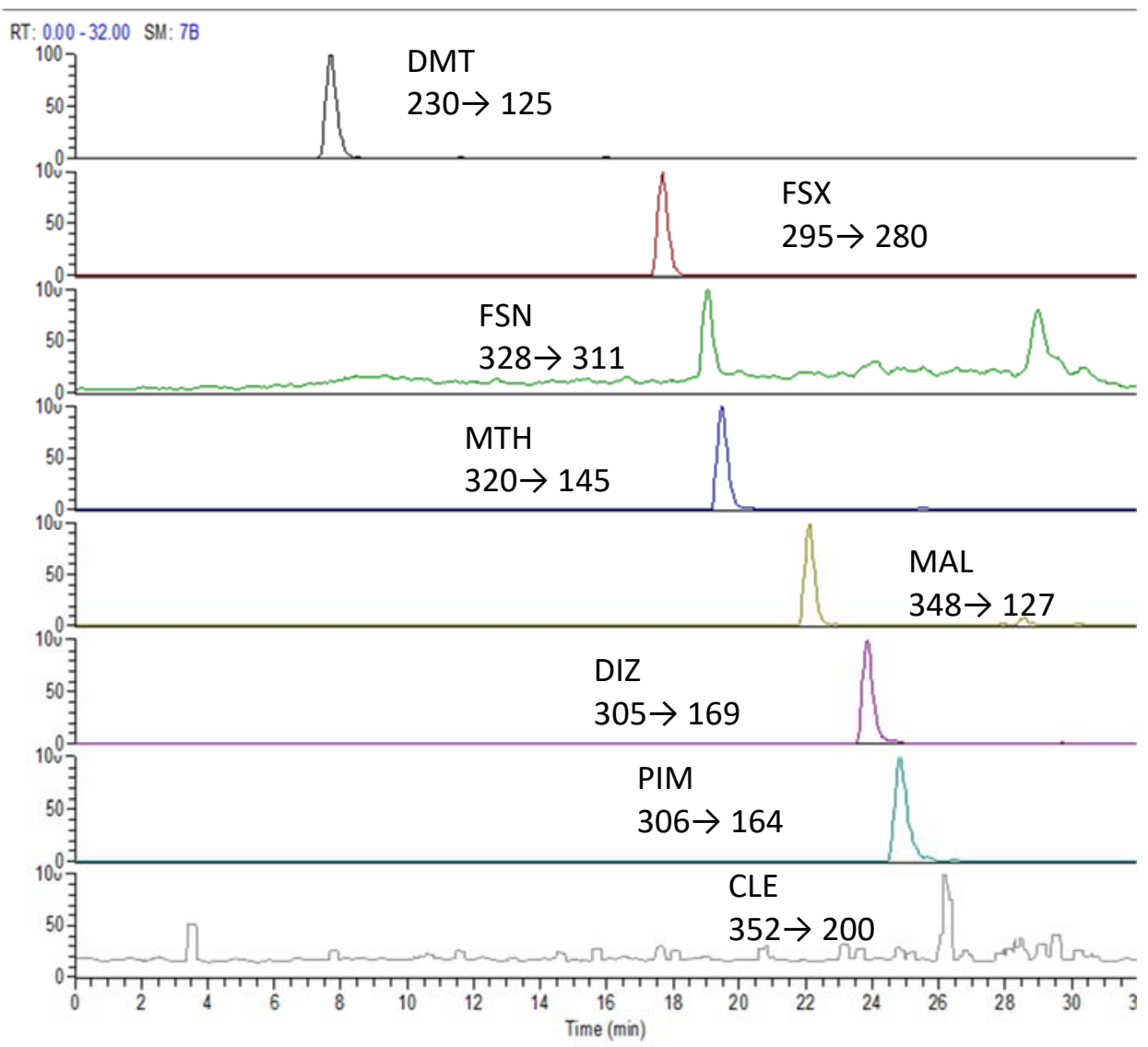

Figure S2: LC-MS chromatograms (MRM mode) of the elution fraction of an almond oil extract spiked at 100 $\mu \mathrm{g} / \mathrm{kg}$ with eight OPs issued of the MIP. Extraction procedure was described in part 2.5.2 (washing volume of $0.65 \mathrm{~mL}$ ). 